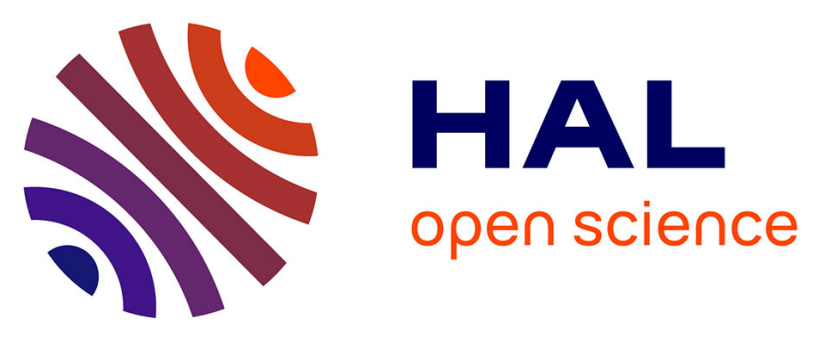

\title{
Electrical study of pentacene-based metal-semiconductor-metal structure: Schottky barrier and active layer thickness effects
}

Wassim Khaldi, Aimen Boubaker, Abdelghaffar Nasri, Kamal Lmimouni, Adel Kalboussi

\section{To cite this version:}

Wassim Khaldi, Aimen Boubaker, Abdelghaffar Nasri, Kamal Lmimouni, Adel Kalboussi. Electrical study of pentacene-based metal-semiconductor-metal structure: Schottky barrier and active layer thickness effects. IEEE Transactions on Electron Devices, 2018, 65 (11), pp.5009-5013. 10.1109/TED.2018.2869537 . hal-03185606

\section{HAL Id: hal-03185606 https://hal.science/hal-03185606}

Submitted on 23 Aug 2021

HAL is a multi-disciplinary open access archive for the deposit and dissemination of scientific research documents, whether they are published or not. The documents may come from teaching and research institutions in France or abroad, or from public or private research centers.
L'archive ouverte pluridisciplinaire HAL, est destinée au dépôt et à la diffusion de documents scientifiques de niveau recherche, publiés ou non, émanant des établissements d'enseignement et de recherche français ou étrangers, des laboratoires publics ou privés.

\section{(c)(1)}

Distributed under a Creative Commons Attribution| 4.0 International License 


\title{
Electrical Study of Pentacene-Based Metal-Semiconductor-Metal Structure: Schottky Barrier and Active Layer Thickness Effects
}

\author{
Wassim Khaldi ${ }^{\circledR}$, Aïmen Boubaker, Abdelghaffar Nasri, Kamal Lmimouni, and Adel Kalboussi
}

\begin{abstract}
The impact of electrodes and active layer thickness on the current-voltage characteristics of Au/Pentacene/Al structure was studied using a physicallybased 2-D simulation by solving Poisson's, continuity, and drift diffusion equations. The main parameters required for simulation are extracted from the logarithmic representation of experimental current-voltage curves. The simulation results produce an excellent overlapping with the experimental data after including parameters previously found in our model. Finally, the simulation was used to better understand the physical processes together with mechanisms governing the efficiency of the device under investigation and to have a predictive behavior.
\end{abstract}

Index Terms-Electronics, modeling, organic, pentacene, Schottky diode.

\section{INTRODUCTION}

O RGANIC thin films, which can be obtained through various techniques, such as molecular beam epitaxy, thermal evaporation, and vacuum sublimation, have numerous advantages over inorganic thin films such as low-temperature process, mechanical flexibility, large-area coverage, low cost, and easy preparation techniques [1], [2].

Pentacene is an organic semiconductor belonging to the polyacenes family that is mostly used as an active layer in the organic device.

As several organic materials, pentacene has attracted considerable attention among researchers in the recent past due to its attractive properties, which make it frequently used in electronics components fabrication, such as organic field effect transistors, photodiodes, organic light-emitting diodes, Schottky barrier diodes, solar cells photovoltaics, and logic

W. Khaldi, A. Boubaker, A. Nasri, and A. Kalboussi are with the Microélectronic and Instrumentation Laboratory, Faculty of Sciences, Monastir University, Monastir 5000, Tunisia (e-mail: wassim.khaldi@ hotmail.fr).

$\mathrm{K}$. Lmimouni is with CNRS, Centrale Lille, ISEN, University of Valenciennes, UMR 8520-IEMN, University of Lille, 59000 Lille, France. gate applications [3]-[9]. Research in organic Schottky structures has received an important attention for many years, and especially in these recent years to develop a new generation of organic Schottky diodes.

Smerdon et al. [10]

fabricated a molecular Schottky diode, based on pentacene and $\mathrm{C} 60$ semiconductors with a rectification ratio $>1000$ at biases of $1 \mathrm{~V}$.

In another work, Kang et al. [11] have fabricated $\mathrm{Au} /$ pentacene/Al with and without pentafluorobenzenethiol (PFBT) self-assembled monolayer (SAM). The PFBT-based SAMs coating on the Au anode.

SAM is thin ordered films of molecules integrated between the active layer and electrode, which provide a good charge injection and reduce the charge traps density [12], [13].

The current density at $3 \mathrm{~V}$ and the cutoff frequency were found to be $100 \mathrm{~A} \mathrm{~cm}^{-3}$ and $1.24 \mathrm{GHz}$, respectively.

In this paper, the 2-D numerical simulation of $\mathrm{Au} /$ pentacene/Al Schottky diode using a physically based device simulator Silvaco-Atlas has been presented.

First, various important physical parameters for pentacene semiconductor have been extracted from the double-logarithmic representation found by Fukuda and Asakawa [14]. Then, based on these previously found parameters, simulated current-voltage characteristics are presented and compared with experimental data [14]. Finally, we report on the effect of electrode and thickness variations on the performance of the considered structure.

\section{DeVice Characteristics}

The device structure under investigation is presented in Fig. 1. It consists of pentacene semiconductor as an active layer inserted between the two metallic electrodes (aluminum and gold).

A dc voltage $\left(V_{A}\right)$, varying from -4 to $4 \mathrm{~V}$, was applied to the anode contact.

The main physical parameters of the pentacene and electrodes contact (gold and aluminum) used for Schottky diode device simulations are listed in Table I.

In Fig. 2, we report the energy band diagram of our structure. The work functions of $\mathrm{Au}$ and $\mathrm{Al}$ contacts are 


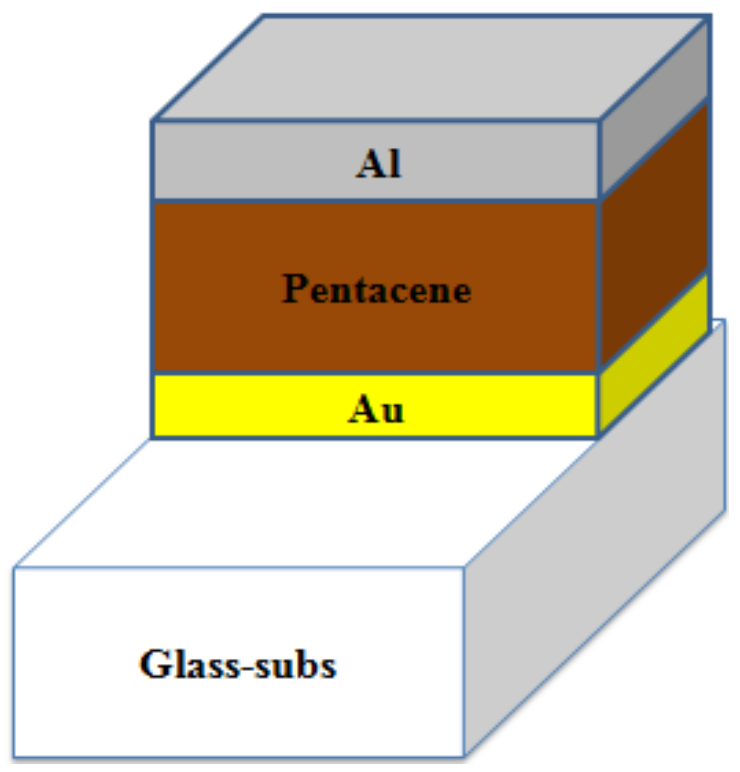

Fig. 1. Schematic of the device structure.

TABLE I

Material Properties of Pentacene, Aluminum, and Gold

\begin{tabular}{cccc}
\hline \hline Materials & Parameter & Value & Unite \\
\hline Pentacene & Affinity & 3 & $\mathrm{eV}$ \\
& Band gap & 2 & $\mathrm{eV}$ \\
& Permittivity & 3 & \\
\multirow{2}{*}{ Aluminum } & Work function & 4.2 & $\mathrm{eV}$ \\
Gold & Work function & 5.1 & $\mathrm{eV}$ \\
\hline \hline
\end{tabular}

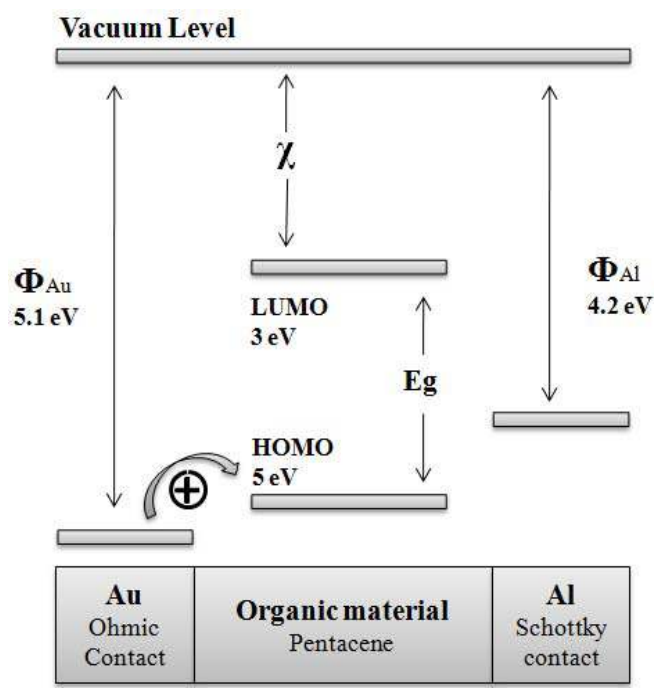

Fig. 2. Energy band diagram.

around 5.1 and $4.2 \mathrm{eV}$, respectively, which are the reported values in [2] and [15]. The pentacene/Al contact establish Schottky barrier, the charge carriers can overcome across just when the forward bias is applied. In the other side, an ohmic contact takes place between gold and pentacene.

Contrary to Schottky contact, the interface of ohmic contact does not present any potential barrier that can prevent the flow of charge carriers, which favors the passage of holes from metal to the semiconductor.

\section{Numerical Simulation}

Characterization and experimental manufactures are very expensive and time-consuming. However, software simulation can be a solution to save time, reduce cost, and find the right solution [16]-[21]. In this paper, there are two variable parameters (Schottky barrier height and thickness) and the studied phenomenon is the effect of these parameters on the current-voltage characteristics.

The 2-D numerical simulations of our devices were performed with physically based device simulator Atlas/Silvaco based on solving systems of coupled differential equations including the drift-diffusion transport model, continuity, and Poisson's equations using finite-element method.

These equations are given as follows:

$$
\begin{aligned}
\operatorname{div}\left(\varepsilon \nabla_{\Psi}\right) & =-\rho \\
\frac{\partial_{n}}{\partial_{t}} & =\frac{1}{q} \operatorname{div} \vec{J}_{n}+G_{n}-R_{n} \\
\frac{\partial_{p}}{\partial_{t}} & =-\frac{1}{q} \operatorname{div} \vec{J}_{p}+G_{p}-R_{p}
\end{aligned}
$$

where $\Psi$ is the electrostatic potential, $\varepsilon$ is the dielectric constant, $\rho$ is the charge distribution, $p$ is the hole density, $n$ is the electron density, $p$ is the holes, $n$ is the electrons, $q$ is the fundamental electronic charge, $G$ is the charge generation rate, $R$ is the charge recombination rate, and $J$ is the current density that is given considering its drift and diffusion components as

$$
\begin{aligned}
& \overrightarrow{J_{n}}=-q n \mu_{n} \vec{E}+q D_{n} \nabla_{n} \\
& \overrightarrow{J_{P}}=-q p \mu_{p} \vec{E}-q D_{p} \nabla_{p}
\end{aligned}
$$

where $\nabla$ is the mathematic operator nabla, $q$ is the fundamental electronic charge, $\mu_{n}$ and $\mu_{p}$ are the electron and hole mobility, respectively, $E$ is the local electric field, $p$ and $n$ are the electron and hole densities, respectively. $D_{n}$ and $D_{p}$ are the Einstein diffusion constants expressed as follows:

$$
\begin{aligned}
& D_{n}=\mu_{n} \frac{k T}{q} \\
& D_{p}=\mu_{p} \frac{k T}{q}
\end{aligned}
$$

where $q$ is the fundamental electronic charge, $\mu_{n}$ and $\mu_{p}$ are the electron and hole mobilities, respectively, $T$ is the temperature and $k$ is Boltzmann's constant.

Finally, the electrical behavior associated with the physical structure under specified biased conditions will be predicted.

\section{Results And Discussion}

In order to relate the observed effects to each other and understand the device response, various experimental characterizations have been carried out. The TCAD process can be very useful in minimizing the experimental and analytical 


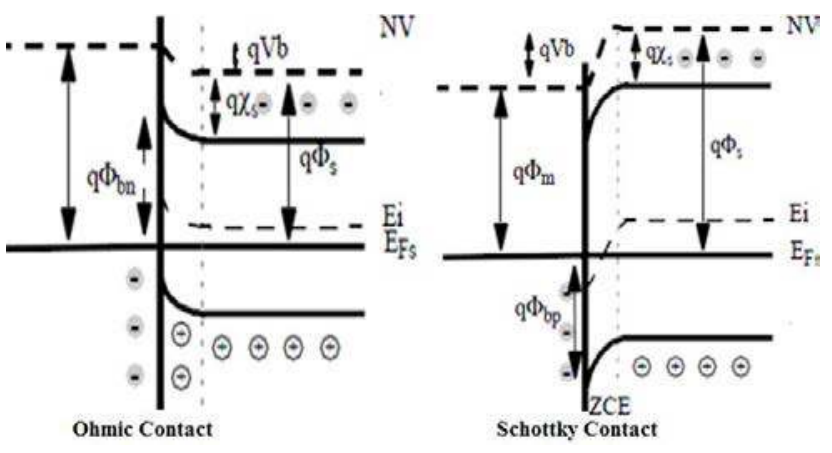

Fig. 3. Interface boundary condition.

modeling efforts because the divers parameters can be modified to clarify the observed phenomenon.

In this section, we used Silvaco TCAD as a semiconductor simulator, which can be adapted to organic devices.

The charge transport mechanisms through pentacene film can be deduced from the analysis of $I-V$ curve. The variation of $\log I$ with $\log V$ for Au/pentacene/Al structure is shown in Fig. 4. The characteristic presents voltage dependence, followed by power law dependence at higher voltage region [14], [22]. This behavior can be accorded to spacecharge-limited current (SCLC). As presented in Fig. 4, four main regions can appear in double-logarithmic characteristic: ohmic, SCLC, trap filling limit, and trap-free SCLC regimes [23], [24].

Traps density, carriers density, and mobility of the active layer (pentacene) were estimated from Fig. 3 by applying the following equations [25], [26].

To calculate the density of trap $\mathrm{Nt}$, we have exploited the Poisson equation

$$
\frac{d E_{\mathrm{FL}}}{d x}=\frac{q \mathrm{Nt}}{\varepsilon \varepsilon_{0}}
$$

where $E_{\mathrm{FL}}$ is the field corresponding to $V_{\mathrm{FL}}$ (the beginning of the regime 4 where all the traps are filled). The integration of this equation provides us $V_{\mathrm{FL}}$ immediately

$$
V_{\mathrm{FL}}=\frac{q \mathrm{Nt} d^{2}}{2 \varepsilon \varepsilon_{0}}
$$

where $d, q$, and $\varepsilon$ are the thickness, the charge, and the permittivity, respectively.

Thus, we can easily deduce the trap concentration $\mathrm{Nt}$

$$
\mathrm{Nt}=\frac{2 \varepsilon \varepsilon_{0} V_{\mathrm{FL}}}{q d^{2}} .
$$

A trap density of about $3.10^{16} \mathrm{~cm}^{-3}$ was obtained for $V_{\mathrm{FL}}=2.2 \mathrm{~V}$ and $d=160 \mathrm{~nm}$. This value is in excellent agreement with many other studies reported in [24] and [27].

The carriers number $n_{0}$ can be obtained from the following expression:

$$
n_{0}=\frac{9 \varepsilon \varepsilon_{0}}{8 q d^{2}} V_{\mathrm{ohm}} .
$$

For $V_{\mathrm{ohm}}=0.42 \mathrm{~V}$ and $d=160 \mathrm{~nm}$, a density of carriers was found in the order of $4 \times 10^{16} \mathrm{~cm}^{-3}$, which is in good agreement with data reported in [28] and [29].

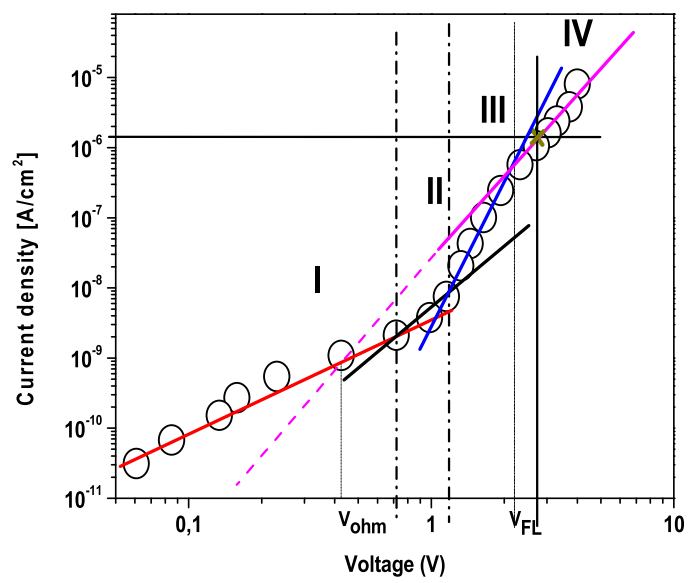

Fig. 4. Double-logarithmic representation of $I-V$ curves.

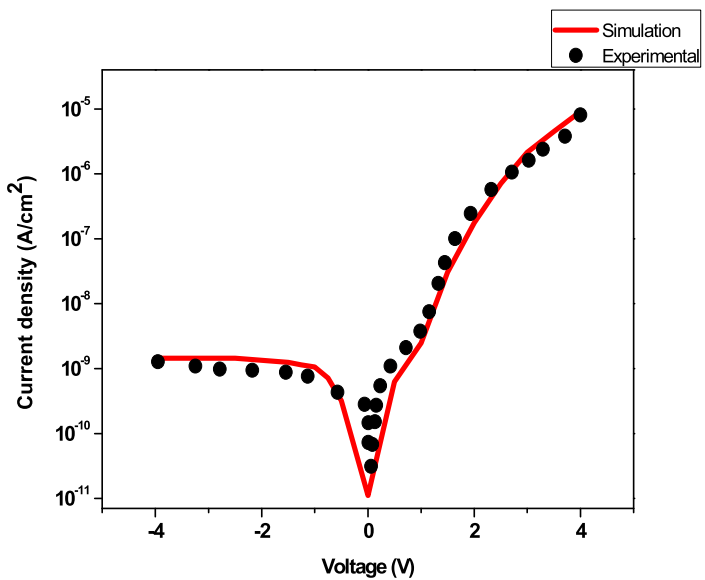

Fig. 5. Comparison between the experimental data [14] (symbol) and simulated (line) $I-V$ curves.

Finally, the mobility was determined from the experimental characteristic in zone IV and presented in the following equation:

$$
J_{\mathrm{scl}}=\frac{9 \varepsilon \varepsilon_{0} \mu V^{2}}{8 d^{3}} .
$$

Hence, we can directly conclude the mobility, from the logarithmic representation of the current density as a function of the voltage for the device under study.

As shown in Fig. 4, for $V=2.6 \mathrm{~V}, I=1.6 \times$ $10^{-6} \mathrm{~A} / \mathrm{cm}^{2}$ which gives a mobility of about $0.39 \times$ $10^{-4} \mathrm{~cm}^{2} \mathrm{v}^{-1} \mathrm{~s}^{-1}$. This value is close to several other experimental results [11], [26], [30].

The current-voltage characteristics obtained on the basis of Atlas simulation are compared with the experimental values reported by others on the same structure and is shown in Fig. 5. As can be seen from this comparison, our simulation results are in good agreement with the experimental data reported in [14], verifying the validity and accuracy of the model and the parameters extracted previously.

In order to understand the influence of the Schottky barrier on the current-voltage characteristic, results of $I-V$ simulations as the function of the electrode (tin, titanum, and aluminum metals [31]) are discussed in the following. 


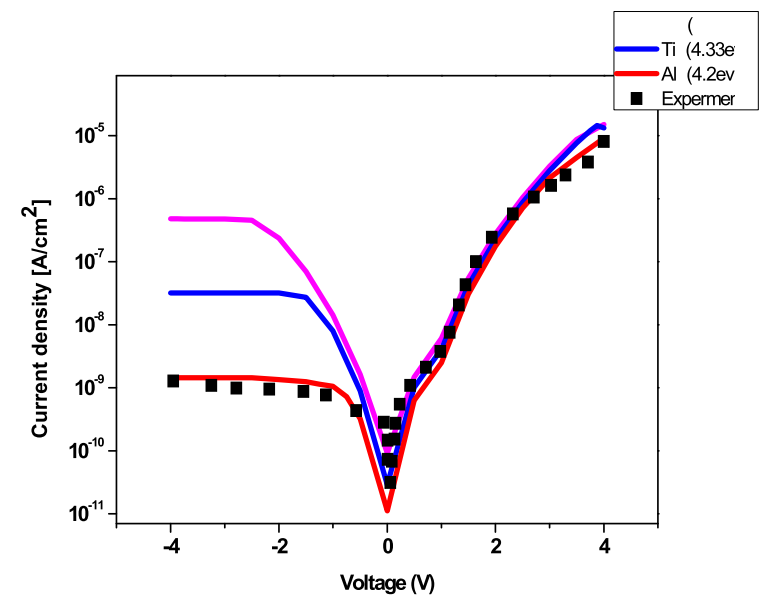

Fig. 6. Effect of electrodes in current-voltage characteristic.

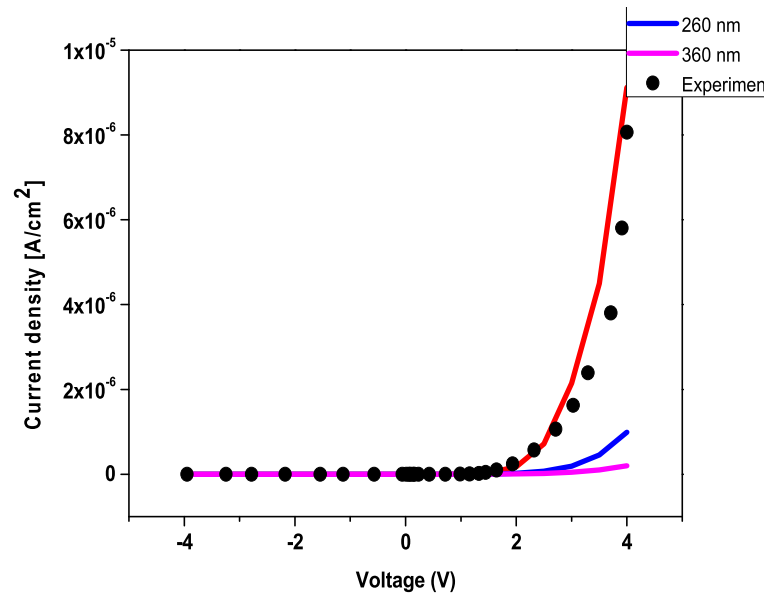

Fig. 7. Influence of active layer thickness.

As shown in Fig. 6, the $I-V$ curves are changed from the typical rectification characteristics to the ohmic behaviors as the electrode change.

Indeed, changing the electrode affects directly the electrical characteristic by modifying the height of the Schottky barrier, which leads to more charge transport from and to the electrode and eventually, the leakage current with the reverse bias increases with the metal work function.

Finally, by comparing the rectification ratio values for each electrode, we can conclude from Fig. 6 that the best one was found for aluminum contact, which has the lowest work function.

The current-voltage characteristics of $\mathrm{Au} /$ pentacene/Al device under forward and reverse voltages at different thickness of the pentacene semiconductor are presented in Fig. 7. The current density increases with the decreasing active layer thickness. Reducing in the resistivity of the pentacene layer with the decrement in the pentacene thickness is the probable reason for this raised value at the current density.

Finally, to verify our results, we compare the previously simulated current values with the values calculated theoretically in (12) for a voltage equal to $4 \mathrm{~V}$.

A comparison between the simulated and analytical current densities for the three active layers thicknesses is
TABLE II

SUMMARY OF MEASUREMENTS: SIMULATED AND THEORETICAL CURRENT DENSITY

\begin{tabular}{ccc}
\hline \hline & $\begin{array}{c}\text { Simulated current } \\
\text { density }\end{array}$ & $\begin{array}{c}\text { Theoretical current } \\
\text { Density } \\
\mathbf{9} \varepsilon_{0} \mu \mathbf{V}^{\mathbf{2}}\end{array}$ \\
\hline $360 \mathrm{~nm}$ & $2.9110^{-7}$ & $\mathbf{J}_{\mathbf{S c l}}=\frac{\mathbf{8 ~ d}^{\mathbf{3}}}{(12)}$ \\
\hline $260 \mathrm{~nm}$ & $1.1110^{-6}$ & $1.9910^{-7}$ \\
$160 \mathrm{~nm}$ & $8.3710^{-6}$ & $7.00410^{-6}$ \\
& & \\
\hline \hline
\end{tabular}

provided in Table II. As expected, all of the theoretical values close to those obtained by the simulation.

\section{CONCLUSION}

In summary, by using a numerical simulation, we have studied the influence of both the active layer thickness and the electrode on the $I-V$ tendency in the Au/pentacene/Al Schottky diode structure.

After extracting the main pentacene parameters necessary for the simulation, from the logarithmic characteristic, a validation with experimental curves was done and a good agreement was observed.

As a result, we have found that the electrical characteristic is influenced by the nature of the electrode that defines the Schottky barrier, which can directly affect the rectification ratio and the leakage current.

This paper presents a new possibility in making the efficient organic diode by testing the structure under investigated and pick out the correct parameters necessary to have a good operation before the fabrication process, which will enormously reduce the component manufacturing cost.

\section{REFERENCES}

[1] N. Oyama, S. Kaneko, K. Momiyama, K. Kanomata, and F. Hirose, "Effects of interfacial oxide layer in P3HT/n-Si organic/inorganic heterojunction diodes on their carrier transport properties," Microelectronic Eng., vol. 104, pp. 130-134, Apr. 2013, doi: 10.1016/j.mee.2012.11.025.

[2] W. Khaldi et al., "Traps density and temperature effects on the performance of organic rectifying diode based on pentacene," Organic Electron., vol. 44, pp. 106-109, May 2017, doi: 10.1016/j.orgel.2017.01.039.

[3] S. Kumar, K. R. Justin Thomas, C.-T. Li, and K.-C. Ho, "Synthesis and photovoltaic properties of organic dyes containing $N$-fluoren-2-yl dithieno[3,2-b:2',3'- $d]$ pyrrole and different donors," Org. Electron., vol. 26, pp. 109-116, Nov. 2015, doi: 10.1016/j.orgel.2015.07.019.

[4] A. Nasri, A. Boubaker, B. Hafsi, W. Khaldi, and A. Kalboussi, "Tuning negative differential resistance in a single molecule transistor: Designs of logic gates and effects of various oxygen- and hydrogeninduced defects," Dig. J. Nanomaterials Biostructures, vol. 12, no. 1, pp. 99-110, 2017.

[5] B. Hafsi, A. Boubaker, D. Guerin, S. Lenfant, A. Kalboussi, and K. Lmimouni, "Traps and interface fixed charge effects on a solutionprocessed $n$-type polymeric-based organic field-effect transistor," J. Electron. Mater, vol. 46, no. 2, pp. 1128-1136, 2017, doi: $10.1007 /$ s11664-016-5067-3.

[6] Y. An et al., "The rectifying effect of heterojunctions composed of carbon and boron nitride nanotubes," Organic Electron., vol. 50, pp. 43-47, Nov. 2017, doi: 10.1016/j.orgel.2017.07.027.

[7] A. Sugimoto, H. Ochi, S. Fujimura, A. Yoshida, T. Miyadera, and M. Tsuchida, "Flexible OLED displays using plastic substrates," IEEE J. Sel. Topics Quantum Electron., vol. 10, no. 1, pp. 107-114, Jan./Feb. 2004, doi: 10.1109/JSTQE.2004.824112. 
[8] S. Ali, J. Bae, and C. H. Lee, "Organic diode with high rectification ratio made of electrohydrodynamic printed organic layers," Electron. Mater. Lett., vol. 12, no. 2, pp. 270-275, 2016, doi: 10.1007/s13391015-5202-y.

[9] A. Nasri, A. Boubaker, B. Hafsi, W. Khaldi, and A. Kalboussi, "A comparison study of electrode material effects on the molecular single electron transistor," Organic Electron., vol. 48, pp. 7-11, Sep. 2017, doi: 10.1016/j.orgel.2017.05.033.

[10] J. A. Smerdon, N. C. Giebink, N. P. Guisinger, P. Darancet, and J. R. Guest, "Large spatially resolved rectification in a donor-acceptor molecular heterojunction," Nano Lett., vol. 16, no. 4, pp. 2603-2607, 2016, doi: 10.1021/acs.nanolett.6b00171.

[11] C.-M. Kang et al., "1 GHz Pentacene diode rectifiers enabled by controlled film deposition on SAM-treated au anodes," Adv. Electron. Mater, vol. 2, no. 2, p. 1500282, 2016, doi: 10.1002/aelm.201500282.

[12] R. Hayakawa, M. Petit, T. Chikyow, and Y. Wakayama, "Interface engineering for molecular alignment and device performance of quaterrylene thin films," Appl. Phys. Lett., vol. 93, no. 15, p. 153301, 2008, doi: 10. $1063 / 1.2998404$

[13] J. P. Hong, A. Y. Park, S. Lee, J. Kang, N. Shin, and D. Y. Yoon, "Tuning of Ag work functions by self-assembled monolayers of aromatic thiols for an efficient hole injection for solution processed triisopropylsilylethynyl pentacene organic thin film transistors," Appl. Phys. Lett., vol. 92, no. 14, p. 143311, 2008, doi: 10.1063/1.2907691.

[14] K. Fukuda and N. Asakawa, "Spin-dependent electrical conduction in a pentacene Schottky diode explored by electrically detected magnetic resonance," J. Phys. D, Appl. Phys., vol. 50, no. 5, p. 055102, 2017, doi: 10.1088/1361-6463/aa524b.

[15] B. N. Pal, J. Sun, B. J. Jung, E. Choi, A. G. Andreou, and H. E. Katz, "Pentacene-zinc oxide vertical diode with compatible grains and 15-MHz rectification," Adv. Mater, vol. 20, no. 5, pp. 1023-1028, Mar. 2008, doi: 10.1002/adma.200701550

[16] P. Lopez-varo, J. A. Jiménez-Tejada, O. Marinov, C. H. Chen, and M. J. Deen, "Charge density at the contacts of symmetric and asymmetric organic diodes," Organic Electron., vol. 35, pp. 74-86, Aug. 2016, doi: 10.1016/j.orgel.2016.05.009.

[17] C. H. Shim, F. Maruoka, and R. Hattori, "Structural analysis on organic thin-film transistor with device simulation," IEEE Trans. Electron Devices, vol. 57, no. 1, pp. 195-200, Jan. 2010, doi: 10.1109/TED.2009. 2035540.

[18] P. Mittal, Y. S. Negi, and R. K. Singh, "An analytical approach for parameter extraction in linear and saturation regions of top and bottom contact organic transistors," J. Comput. Electron., vol. 14, no. 3, pp. 828-843, 2015, doi: 10.1007/s10825-015-0719-8.

[19] N. Matsuo, T. Kobayashi, and A. Heya, "Improvement of conversion efficiency for solar cell with metal-oxide-semiconductor diode," Electron. Lett., vol. 49, no. 21, pp. 1351-1353, Oct. 2013, doi: 10.1049/el.2013.2485.

[20] S. Hlali, N. Hizem, and A. Kalboussi, "High- $k$ dielectric materials for the gate oxide of a MIS capacitor: Effect of interface states on the C-V characteristics," J. Comput. Electron., vol. 15, no. 4, pp. 1340-1350, 2016, doi: 10.1007/s10825-016-0916-0.

[21] M. Baudrit and C. Algora, "Tunnel diode modeling, including nonlocal trap-assisted tunneling: a focus on III-V multijunction solar cell simulation," IEEE Trans. Electron Devices, vol. 57, no. 10, pp. 2564-2571, Oct. 2010, doi: 10.1109/TED.2010.2061771.

[22] J. Lee, S. S. Kim, K. Kim, J. H. Kim, and S. Im, "Correlation between photoelectric and optical absorption spectra of thermally evaporated pentacene films," Appl. Phys. Lett., vol. 84, no. 10, p. 1701, 2004, doi: 10.1063/1.1668328

[23] I. Missoum, Y. S. Ocak, M. Benhaliliba, C. E. Benouis, and A. Chaker, "Microelectronic properties of organic Schottky diodes based on MgPc for solar cell applications," Synth. Met., vol. 214, pp. 76-81, Apr. 2016, doi: 10.1016/j.synthmet.2016.01.004.

[24] C. Voz et al., "Optoelectronic devices based on evaporated pentacene films," Sol. Energy Mater. Sol. Cells, vol. 87, nos. 1-4, pp. 567-573, 2005, doi: 10.1016/j.solmat.2004.07.039.

[25] A. Moliton, W. Rammal, and B. Lucas, "A new method for the determination of electronic mobility in organic materials associated with optoelectronic devices," EPL (Europhysics Lett.), vol. 72, no. 5, pp. 754-759, 2005, doi: 10.1209/epl/i2005-10311-0.

[26] B. Lucas, A. El Amrani, A. Moliton, A. Skaiky, A. El Hajj, and M. Aldissi, "Charge transport properties in pentacene films: Evaluation of carrier mobility by different techniques," Solid-State Electron., vol. 69, pp. 99-103, Mar. 2012, doi: 10.1016/j.sse.2011.12.011.
[27] A. Benor, A. Hoppe, V. Wagner, and D. Knipp, "Electrical stability of pentacene thin film transistors," Organic Electron., vol. 8, no. 6, pp. 749-758, 2007, doi: 10.1016/j.orgel.2007.06.005.

[28] Y. S. Yang and T. Zyung, "Preparation and electronic defect characteristics of pentacene organic field effect transistors," Macromolecular Res., vol. 10, no. 2, pp. 75-79, Mar. 2002, doi: 10.1007/BF03218293.

[29] W. T. Wondmagegn et al., "Experimental and modeling study of the capacitance-voltage characteristics of metal-insulator-semiconductor capacitor based on pentacene/parylene," Thin Solid Films, vol. 519, no. 13, pp. 4313-4318, 2011, doi: 10.1016/j.tsf.2011.02.014.

[30] R. Scholz et al., "CComparison between the charge carrier mobilities in pentacene OFET structures as obtained from electrical characterization and potentiometry," Proc. SPIE, vol. 5940, p. 59400I, Sep. 2005, doi: 10.1117/12.617004.

[31] S. P. Lee, "Electrodes for semiconductor gas sensors," Sensors, vol. 17, no. 4, p. 683, 2017, doi: 10.3390/s17040683. 